\title{
Myotubular myopathy caused by multiple abnormal splicing variants in the MTM1 RNA in a patient with a mild phenotype
}

\author{
Nasim Vasli ${ }^{1,2,3,4}$, Vincent Laugel ${ }^{5}$, Johann Böhm ${ }^{1,2,3,4}$, Béatrice Lannes ${ }^{6}$, Valérie Biancalana ${ }^{1,2,3,4,7}$ and \\ Jocelyn Laporte L, $, 2,3,4^{-1}$
}

Mutations impacting on the splicing of pre-mRNA are one important cause of genetically inherited diseases. However, detection of splice mutations, that are mainly due to intronic variations, and characterization of their effects are usually not performed as a first approach during genetic diagnosis. X-linked recessive myotubular myopathy is a severe congenital myopathy due to mutations in the MTM1 gene encoding myotubularin. Here, we screened a male patient showing an unusually mild phenotype without respiratory distress by western blot with specific myotubularin antibodies and detected a strong reduction of the protein level.The disease was subsequently linked to a hemizygous point mutation affecting the acceptor splice site of exon 8 of $M T M 1$, proven by protein, transcript and genomic DNA analysis. Detailed analysis of the MTM1 mRNA by RT-PCR, sequencing and quantitative PCR revealed multiple abnormal transcripts with retention of a truncated exon 8 , and neighboring exons 7 and 9 but exclusion of several other exons, suggesting a complex effect of this mutation on the splicing of non-adjacent exons. We conclude that the analysis of RNA by RT-PCR and sequencing is an important step to characterize the precise impact of detected splice variants. It is likely that complex splice aberrations due to a single mutation also account for unsolved cases in other diseases.

European Journal of Human Genetics (2012) 20, 701-704; doi:10.1038/ejhg.2011.256; published online 18 January 2012

Keywords: splice mutation; MTM1; myotubular myopathy; centronuclear myopathy; myotubularin; congenital myopathy

\section{INTRODUCTION}

Centronuclear myopathies are rare congenital myopathies with $\mathrm{X}$-linked and autosomal inheritance. X-linked recessive myotubular myopathy (XLMTM; OMIM \# 310400) is the most severe form, characterized by severe hypotonia at birth and respiratory insufficiency associated with a poor prognosis. Most patients have a short life span and harbor mutations in the MTM1 gene encoding the phosphoinositide phosphatase myotubularin. ${ }^{1,2}$

To date, molecular diagnosis of about 400 patients with MTM1 mutation have been reported, mainly based on direct sequencing of exons and intron-exon junctions., ${ }^{3,4}$ Some of the mutations were found to affect the canonical acceptor (AG) or donor (GT) splice sites, involving alteration or skipping of the mutated exon but an impact on other exons has not been reported. ${ }^{5-7}$

Here, we report the detection and characterization of a novel mutation affecting the acceptor splice site of exon 8 in the MTM1 gene, inducing splicing defects of non-adjacent exons, and leading to multiple aberrant transcripts.

\section{PATIENTS AND METHODS}

Patient

The male patient was born at 38-weeks' gestation. Fetal movements were decreased during pregnancy. He required oxygen administration in the first $2 \mathrm{~h}$ of life but did not need endotracheal intubation or mechanical ventilation. Major general hypotonia was present from birth with a narrow and elongated face, high-arched palate, decreased spontaneous movements, weak grasping reflex, weak cry and poor sucking. Left congenital torticollis and mild knee contractures were also present from birth. Motor milestones were delayed and he needed continued physiotherapy. He had numerous mild respiratory infections during the first 2 years although no sign of respiratory distress was ever noted. At 5 years of age, generalized muscle weakness is still present and facial weakness with dysarthria and chewing difficulties are still prominent. Muscle biopsy (quadriceps) showed the typical centronuclear myopathies pattern of small rounded muscle fibers with central nuclei (Figure 1).

\section{Molecular biology procedures}

Genomic DNA of the patient and his mother was isolated from blood samples or cultured lymphoblastoid cells using standard procedures. Molecular investigations were performed with the informed consent of the family.

For western blot, myotubularin was detected with the C-terminal R2827 anti-myotubularin polyclonal antibody as described. ${ }^{8}$ Equal loading was monitored by Coomassie staining and a mouse monoclonal anti-GAPDH antibody (Millipore, Billerica, MA, USA).

Total RNA was extracted from lymphoblastoid cells or muscle using Tri reagent (Molecular Research Center Inc., Cincinnati, OH, USA),

${ }^{1}$ Department of Translational Medicine and Neurogenetics, IGBMC (Institut de Génétique et de Biologie Moléculaire et Cellulaire), IIIkirch, France; ${ }^{2}$ Inserm, U964, IIIkirch, France; ${ }^{3}$ CNRS, UMR7104, Illkirch, France; ${ }^{4}$ University of Strasbourg, Illkirch, France; ${ }^{5}$ Department of Pediatrics, Strasbourg University Hospital, Strasbourg, France; ${ }^{6}$ Department of Pathology, Strasbourg University Hospital, Strasbourg, France; ${ }^{7}$ Genetic diagnostic laboratory, Faculty of Medicine, CHRU, Strasbourg, France

*Correspondence: Dr J Laporte, Department of Translational Medicine and Neurogenetics, IGBMC (Institut de Génétique et de Biologie Moléculaire et Cellulaire), 1 rue Laurent Fries, B.P. 10142, 67404 Illkirch, France. Tel: +33 388653412; Fax: +33 388653201. E-mail: jocelyn@igbmc.fr

Received 5 August 2011; revised 6 December 2011; accepted 7 December 2011; published online 18 January 2012 

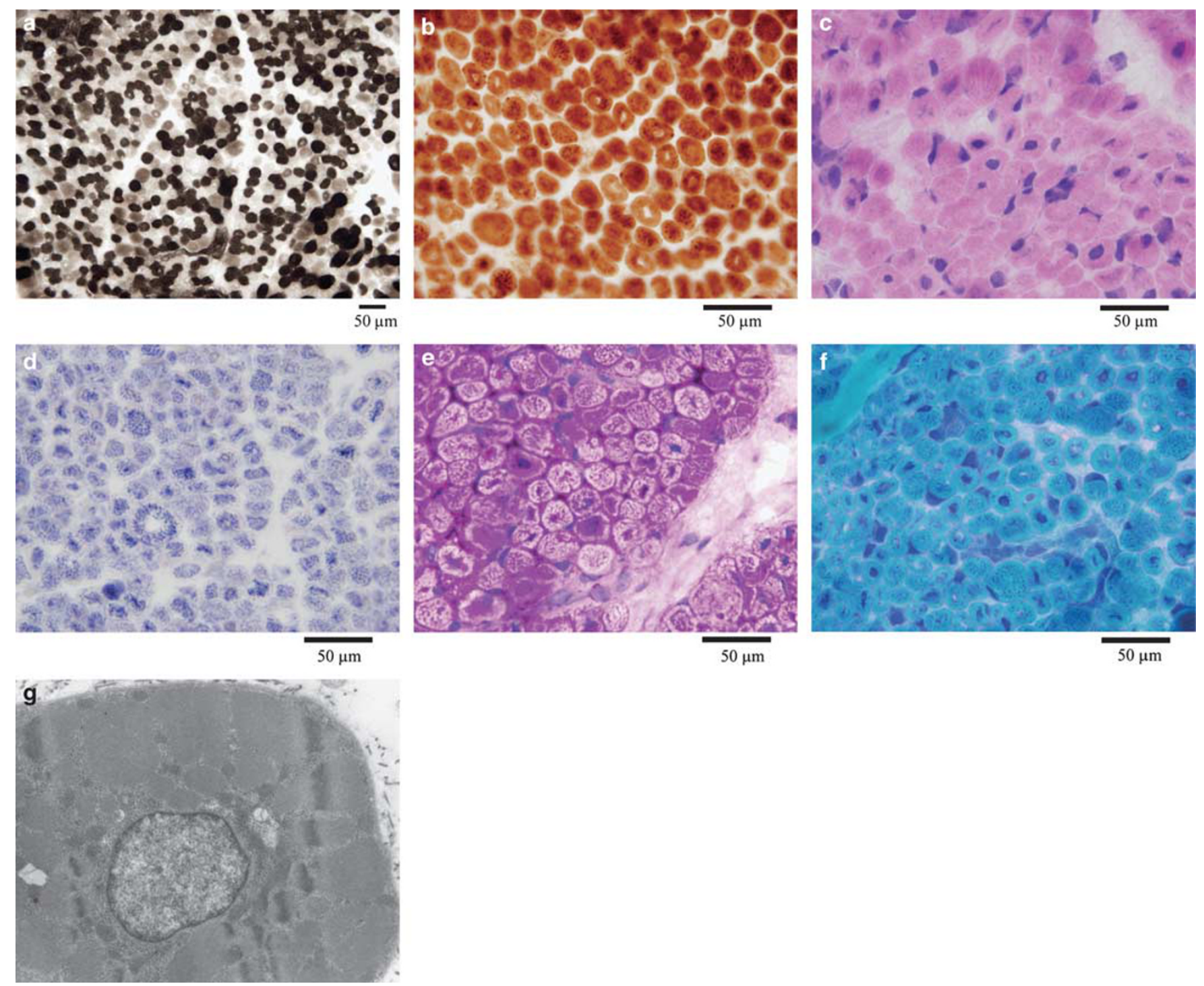

Figure 1 Histological analysis of the patient's muscle biopsy stained with ATPase, $\mathrm{pH}=4.5$ (a), Cytochrome oxidase (cox) (b), Haematoxylin and Eosin (c), Nicotinamide adenosine dinucleotide - tetrazolium reductase (NADH-TR) (d) Periodic Acid Schiff (PAS) (e) and Gomori Trichrome (f). (g) Electron microscopic picture shows muscle fibers with a centralized nucleus surrounded by an area devoid of myofibrils and filled with glycogen granules. Myofibrils are of different size.

reverse transcribed and amplified using MTM1 specific primers in three overlapping fragments covering the entire MTM1 coding sequence, as previously reported. ${ }^{8}$ Resulting cDNAs were cloned into the pGEM-T Easy vector (Promega, Madison, WI, USA) and sequenced (GATC; http://www.gatc-biotech.com/). Real-time RT-PCR was performed using a Lightcycler 480 (Roche Diagnostics, Meylan, France) with primers in the ubiquitous exons 12 and 14 and RPLPO and GAPDH mRNAs as standards. All exons and intron-exon boundaries of MTM1, BIN1 and DNM2 were sequenced from genomic DNA. Primer sequences are available upon request.

\section{RESULTS}

Unlike most XLMTM patients, our patient (ACJ7) showed a mild disease progression and no respiratory distress. To assess whether he has XLMTM or a milder autosomal form, and as a first and cost-effective diagnostic approach, we performed western blot on a muscle biopsy sample and lymphoblasts. The amount of MTM1 protein was below the detection level in the patient's lymphoblasts as compared with control cells (Figure 2a), indicating myotubularin deficiency as the disease cause. To decipher whether the diseaseassociated mutation is in the MTM1 gene or in other genes regulating MTM1 transcription, we analyzed the RNA of MTM1 extracted from lymphoblastoid cells and a muscle biopsy. RT-PCR from lymphoblasts revealed the presence of abnormal RNA molecules with variable length of the 5' region (Figure 2b). We sequenced 68 and $56 \mathrm{cDNA}$ clones extracted from the patient lymphoblastoid cells and muscle biopsy, respectively. Sanger sequencing identified five different frequent aberrant transcripts in lymphoblasts cDNA and five aberrant transcripts in muscle cDNA, compared with 50 independent cDNA clones sequenced from lymphoblasts of a healthy control (Figure 2c). We also detected other aberrant transcripts in single clone (data not shown) as well as the normal MTM1 transcript (18 clones from lymphoblast cDNA and 12 from muscle cDNA). All aberrant clones had an abnormal 5' part of exon 8, starting at cryptic acceptor sites at 
a

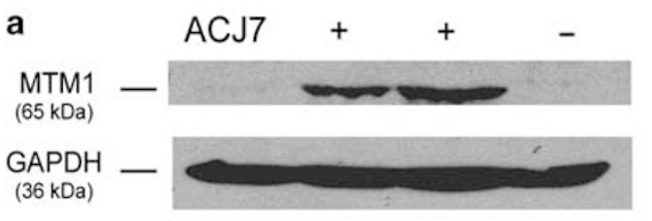

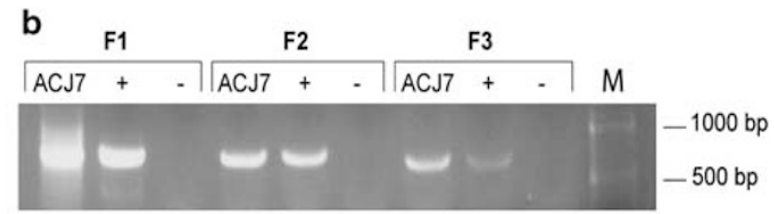
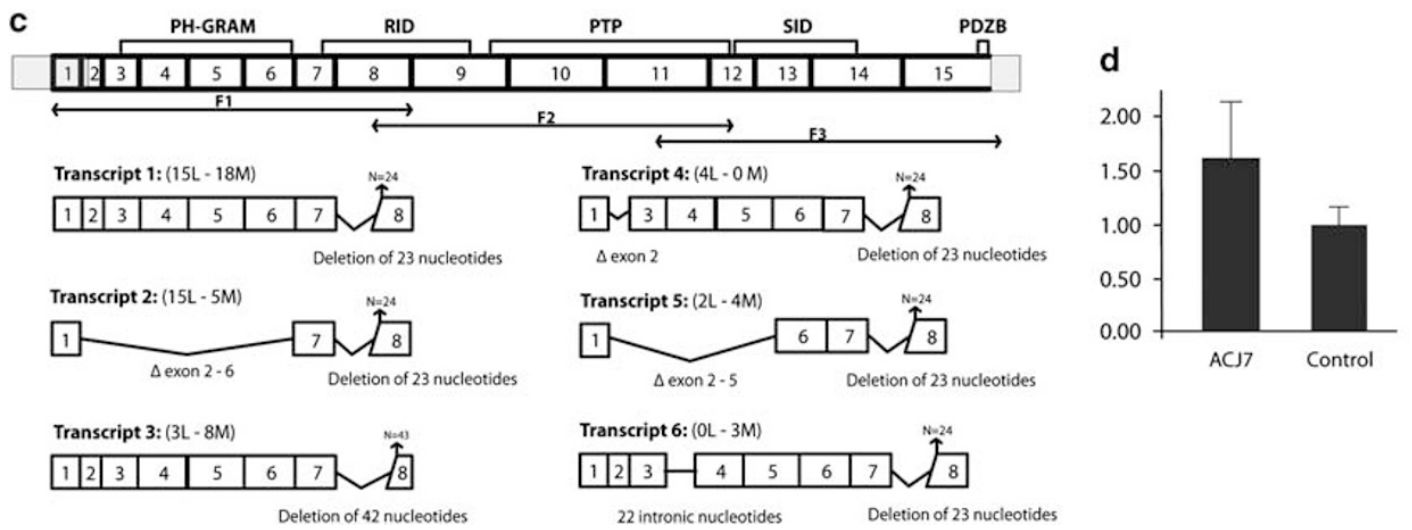

Figure 2 Detection and characterization of the MTM1 splice mutation. (a) Western blot to asses the myotubularin level in cells from the patient (ACJ7), positive (+, cells from a healthy individual) and negative (-, cells from a XLMTM patient with an entire MTM1 gene deletion) controls. (b) RT-PCR covering the MTM1 coding sequence was performed in three overlapping fragments (F1: exons 1-8; F2: exons 8-12; F3: exons 11-15) using RNA extracts from the patient's lymphoblastoid cells and controls. (c) The MTM1 gene has 15 exons drawn to scale with start and stop codons in exon 2 and 15, respectively. The five protein domains are: PH-GRAM (Pleckstrin homology, Glucosyltransferase, Rab-like GTPase activators and Myotubularins), RID (Rac-induced recruitment domain), PTP (Protein Tyrosine Phosphatase-like), SID (SET-interacting domain) and PDZB (PSD-95/Dlg/ZO-1 binding site). F1, F2 and F3 indicate the overlapping PCR fragments in (b). The abnormal transcripts and number of cDNA clones are shown below. $L$ indicates the number of lymphoblastoid clones and $\mathrm{M}$ indicates the number of muscle clones. (d) The amount of MTM1 RNA is not significantly different between ACJ7 patient cells and three controls as seen by quantitative RT-PCR in three independent experiments, using RPLO and GAPDH as standard genes. $2 \triangle \mathrm{Ct}$ method; $P$-value=0.134.

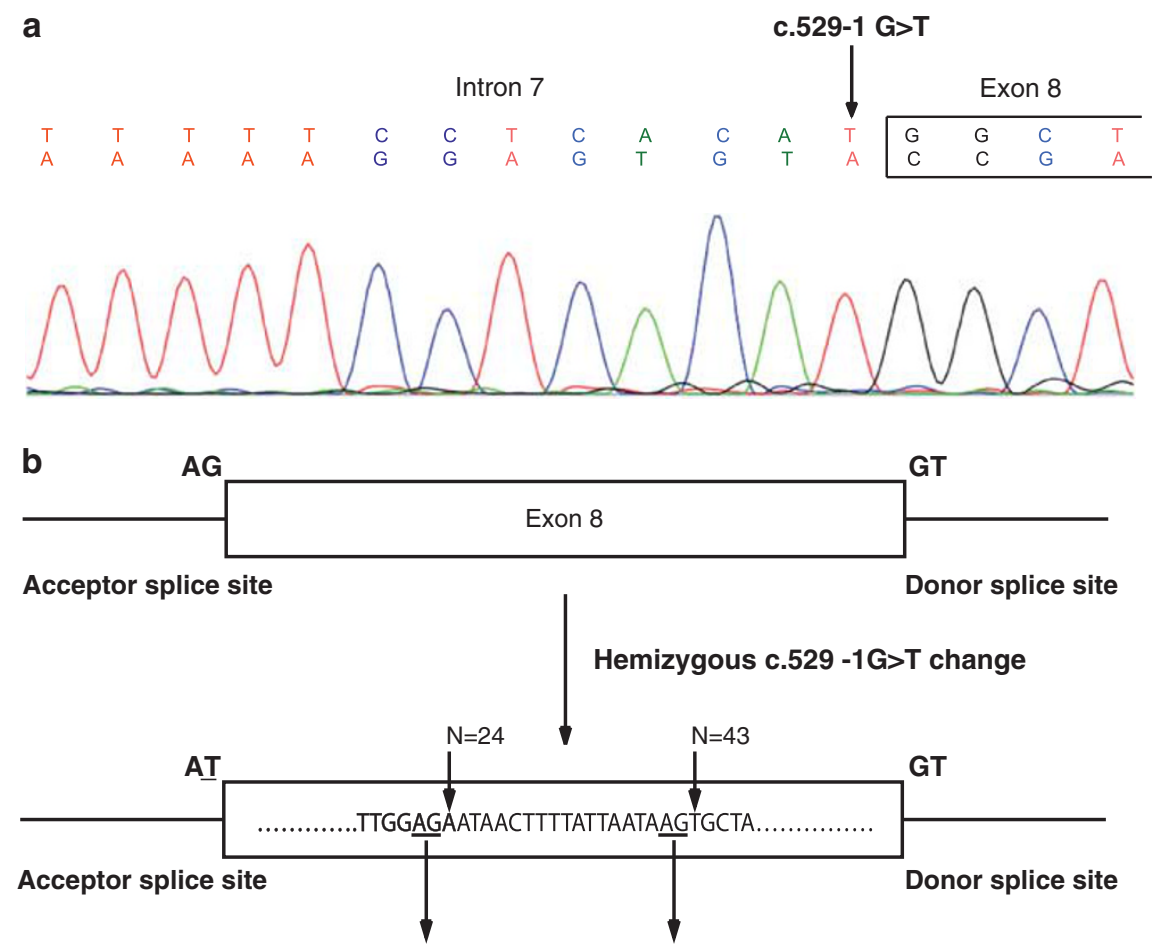

New Cryptic Acceptor splice sites

Figure 3 Genomic mutation and mechanism. (a) Electropherogram of the hemizygous c.529-1G>T change in intron 7 of MTM1 (GenBank U46024). (b) Schematic representation of normal MTM1 exon 8 splice sites and the cryptic acceptor splice sites found to be alternatively used in cells from the patient. 
exon 8 positions 2, 24 (highly preferred) and 43, predicted by the human splice site finder program. ${ }^{9}$ This suggested a mutation in the endogenous acceptor site of exon 8. Unexpectedly, most cDNA molecules displayed aberrant exclusion of non-adjacent exons but all retained exons 1 and 7 .

The detected abnormal MTM1 trancripts in our patient are not due to an overall decrease in RNA level and the amplification of illegitimate splicing background, as quantitative RT-PCR revealed a normal level of MTM1 RNA (Figure 2d).

In accordance to the strong decrease in MTM1 protein and the aberrant splicing variants detected by RT-PCR, sequencing of the patient's genomic DNA revealed a novel hemizygous c.529-1G $>\mathrm{T}$ mutation modifying the physiological acceptor splice site from AG to AT in intron 7, leading to the use of cryptic acceptor splice sites in exon 8 (Figures $3 \mathrm{a}$ and $\mathrm{b}$ ). The mother of the patient is a healthy heterozygous carrier of the mutation.

\section{DISCUSSION}

We report the first MTM1 mutation leading to complex splicing rearrangements in a patient with mildly progressive myotubular myopathy.

Mature mRNAs are assembled by correct identification and joining of exons. The inaccurate attachement of exons leads to the generation of aberrant mRNAs, and unstable, truncated or deleterious proteins. ${ }^{10}$ To date, some MTM1 mutations were shown to have an impact on splicing and the majority of patients with splice site mutations presented with a severe phenotype, although a few cases show mild or moderate phenotypes. ${ }^{4-7,11}$ In muscle and lymphoblastoid cells from the patient described in the present study, the majority of the analyzed MTM1 cDNAs is predicted to lead either to the absence of the starting methionine in exon 2 or to the disruption of the coding frame. Indeed MTM1 protein level was strongly decreased although a weak normal sized band was detected when overloading the patient sample (not shown), probably sustaining the mild phenotype. Systematic analysis of the protein level and transcript integrity as a first screening, is a suitable, fast, reliable and cost-effective diagnostic approach if cultured cells or tissues are available, as most MTM1 mutations lead to a detectable decrease in the protein level. ${ }^{8,12}$ In addition, sequencing the RNA species is seldom performed but the present example shows that it represents a necessary approach to characterize the impact of the causative mutation.

In the analyzed patient, the acceptor site mutation in exon 8 was linked to variable exclusion of non-adjacent exons 2 to 6 , whereas the total level of MTM1 RNA was not compromised. Although unlikely, we cannot exclude the possibility of a second intronic variation that can induce this phenomenon. It is not known how the splice site mutation can have an impact on the inclusion/exclusion of nonadjacent exons. Similarly, the presence of multiple abnormal splicing variants in the CSA gene has been previously reported in a patient with Cockayne syndrome, although the causative mutation was not identified in that case. ${ }^{13}$ A number of diseases associated with mutations that affect pre-mRNA splicing have been described before, ${ }^{14,15}$ including intronic mutations leading to inclusion of cryptic exons. ${ }^{8,16}$

We conclude that the analysis of RNA by RT-PCR and sequencing is an important step to characterize the precise impact of detected splice variants, and in some cases to detect deep intronic mutation. Similar complex pathogenic splicing is expected to exist in most genetic diseases.

\section{CONFLICT OF INTEREST}

The authors declare no conflict of interest.

\section{ACKNOWLEDGEMENTS}

The authors thank Charlotte Fugier and Nicolas Dondaine for technical assistance, Jean-Louis Mandel for support and the DNA and Cell Bank of Généthon (Evry, France) for cell lines. This study was supported by INSERM, CNRS, University of Strasbourg, Collège de France, and by grants from Association Française contre les Myopathies. JB was supported by the Deutsche Forschungsgemeinschaft (DFG).

1 Jungbluth $\mathrm{H}$, Wallgren-Pettersson $\mathrm{C}$, Laporte $\mathrm{J}$ : Centronuclear (myotubular) myopathy. Orphanet J Rare Dis 2008; 3: 26.

2 Laporte J, Hu LJ, Kretz C et al: A gene mutated in X-linked myotubular myopathy defines a new putative tyrosine phosphatase family conserved in yeast. Nat Genet 1996; 13: 175-182.

3 Laporte J, Biancalana V, Tanner SM et al: MTM1 mutations in X-linked myotubular myopathy. Hum Mutat 2000; 15: 393-409.

4 Tsai TC, Horinouchi H, Noguchi S et al: Characterization of MTM1 mutations in 31 Japanese families with myotubular myopathy, including a patient carrying $240 \mathrm{~kb}$ deletion in Xq28 without male hypogenitalism. Neuromuscul Disord 2005; 15: 245-252.

5 Nishino I, Minami N, Kobayashi 0 et al: MTM1 gene mutations in Japanese patients with the severe infantile form of myotubular myopathy. NeuromusculDisord 1998; 8: 453-458.

6 Tanner SM, Laporte J, Guiraud-Chaumeil C, Liechti-Gallati S: Confirmation of prenatal diagnosis results of $\mathrm{X}$-linked recessive myotubular myopathy by mutational screening, and description of three new mutations in the MTM1 gene. Hum Mutat 1998; 11: 62-68.

7 Tanner SM, Schneider V, Thomas NS, Clarke A, Lazarou L, Liechti-Gallati S: Characterization of 34 novel and six known MTM1 gene mutations in 47 unrelated X-linked myotubular myopathy patients. Neuromuscul Disord 1999; 9: 41-49.

8 Tosch V, Vasli N, Kretz C et al: Novel molecular diagnostic approaches for X-linked centronuclear (myotubular) myopathy reveal intronic mutations. Neuromuscul Disord 2010; 20: 375-381.

9 Desmet FO, Hamroun D, Lalande M, Collod-Beroud G, Claustres M, Beroud C: Human Splicing Finder: an online bioinformatics tool to predict splicing signals. Nucleic Acids Res 2009; 37: e67.

10 Cartegni L, Chew SL, Krainer AR: Listening to silence and understanding nonsense: exonic mutations that affect splicing. Nat Rev Genet 2002; 3: 285-298.

11 McEntagart M, Parsons G, Buj-Bello A et al: Genotype-phenotype correlations in X-linked myotubular myopathy. Neuromuscul Disord 2002; 12: 939-946.

12 Laporte J, Kress W, Mandel JL: Diagnosis of X-linked myotubular myopathy by detection of myotubularin. Ann Neurol 2001; 50: 42-46.

13 Komatsu A, Suzuki S, Inagaki T, Yamashita K, Hashizume K: A kindred with Cockayne syndrome caused by multiple splicing variants of the CSA gene. Am J Med Genet A 2004; 128A: 67-71.

14 Nissim-Rafinia M, Kerem B: Splicing regulation as a potential genetic modifier. Trends Genet 2002; 18: 123-127.

15 Targovnik HM, Edouard T, Varela V et al: Two novel mutations in the thyroglobulin gene as cause of congenital hypothyroidism: identification a cryptic donor splice site in the exon 19. Mol Cell Endocrinol 2012; 348: 313-321.

16 Aminoff M, Carter JE, Chadwick RB et al: Mutations in CUBN, encoding the intrinsic factor-vitamin B12 receptor, cubilin, cause hereditary megaloblastic anaemia 1. Nat Genet 1999; 21: 309-313. 